ORIGINAL ARTICLE

\title{
Upper gastrointestinal cancer pathology reporting: a regional audit to compare standards with minimum datasets
}

\author{
P M King, J M Blazeby, J Gupta, D Alderson, M Moorghen
}

J Clin Pathol 2004;57:702-705. doi: 10.1136/icp.2003.013326

See end of article for
authors' affiliations
......................
Correspondence to:
Dr M Moorghen,
Department of
Histopathology, United
Bristol NHS Trust,
Marlborough Street, Bristol
BS2 8HW, UK
m.moorghen@bristol.ac.uk
Accepted for publication
8 December 2003

\begin{abstract}
Aims: Accurate pathological (pTNM) staging of oesophageal and gastric cancer provides important prognostic information. The aim of this study was to compare the standard of pathology reporting of oesophageal and gastric cancer resections from a cancer network with standards set by the Royal College of Pathologists.

Methods: All reports for oesophageal and gastric cancer resections from the five hospitals in the cancer network in 2001 were collected. Individual items of information were compared with minimum datasets provided by the Royal College of Pathologists. Items were classified as "complete", "partially complete", or "absent".

Results: One hundred and ten reports were audited (54 oesophageal and 56 gastric). Fourteen gastric and 17 oesophagectomy reports were over $75 \%$ complete. Clinically important missing data occurred most frequently for the $\mathrm{pM}$ component of TNM staging (pMx omitted in 87 reports) and completeness of resection expressed as a bold statement (absent in 50 reports). Twelve reports could not be classified because the specimen contained no residual tumour after neoadjuvant treatment.

Conclusion: The use of a standard proforma for reporting upper gastrointestinal cancers based on a minimum dataset provided by the Royal College of Pathologists is recommended, with modifications to allow for specimens with no tumour after neoadjuvant treatment.
\end{abstract}

$\mathrm{T}$ he histopathology report provides information that facilitates accurate staging of patients with gastric and oesophageal cancer. The prognosis remains poor for these cancers and an accurate histopathology report is crucial in providing information regarding prognostic factors and possible further treatment. The number and location of involved lymph nodes, involvement of the margins, TNM stage, and response to preoperative neoadjuvant treatment are particularly important. In addition, the histopathology report provides feedback regarding the accuracy of preoperative staging techniques, and can be a useful tool in research and audit, particularly with regard to advances in neoadjuvant treatments.

"The prognosis remains poor for gastric and oesophageal cancers and an accurate histopathology report is crucial in providing information regarding prognostic factors and possible further treatment ${ }^{\prime \prime}$

The recent development of cancer networks for upper gastrointestinal cancer necessitates a standard approach to pathology reporting. A need for consistent reporting has been highlighted in previous audits. An audit of colorectal reporting in Wales in 1997 found that only 78\% of colonic and $47 \%$ of rectal reports met their predetermined minimum standards. ${ }^{1}$ An audit of oesophageal and gastric cancer found similar inconsistencies, with only $77 \%$ of gastric reports and $53 \%$ of oesophageal reports meeting minimum standards. ${ }^{2}$ Both of these audits concluded that there was a need for proforma reporting. The Royal College of Pathologists has provided minimum datasets for the reporting of all common cancers. ${ }^{3}$

The aim of this audit was to compare the standard of pathology reporting for gastric and oesophageal resection specimens with minimum datasets provided by the Royal College of Pathologists.

\section{METHODS}

Five hospitals within the Avon, Wiltshire, and Somerset cancer network agreed to participate in the audit. All pathology reports for gastric and oesophageal cancer resection specimens for the whole of 2001 were assessed. Reports were identified retrospectively using the SNOMED coding system and copies of all reports were obtained and anonymised. Tumours deemed to be junctional after review of the pathology reports were placed in the oesophageal category. All reports were read by a single data collector (PK) and compared with the minimum datasets provided by the Royal College of Pathologists. The category headings in table 1 were taken from the minimum datasets, and reports were scrutinised for the presence or absence of information under each category. Each category on the pathology report was entered on to a database as "complete", "partially complete", or " absent", depending on how much of the information required by the minimum dataset was present within the report. For categories such as "length of specimen" to obtain a complete score all the associated specific parameters had to be present. For example, in the gastric reports, the length of the specimen had to include the greater and lesser curve lengths, in addition to the length of the oesophagus and duodenum if applicable. The tumour size had to include length, width, and depth to obtain a complete score. With regard to pTNM staging, the minimum dataset makes it a requirement for a number or letter (as appropriate) to be ascribed to each of the three components of the pTNM stage. Where distant metastasis cannot be assessed owing to the nature of the pathological specimen, the pM staging category is taken as being $\mathrm{pMx}$.

To validate the accuracy of data collection, 20 reports were selected at random and analysed in the same way by a consultant surgeon (JMB) and a specialist registrar in pathology $(\mathrm{JG})$. Any interpretative differences were discussed at a meeting with a consultant histopathologist (MM) and the reports re-read by the original data collector, taking account of the points raised in the discussion. 
Table 1 Categories present on the minimum datasets

\begin{tabular}{ll}
\hline Categories for gastric reports & Categories for oesophageal reports \\
\hline Personal details & Personal details \\
Type of specimen & Gross description \\
Length of specimen & Tumour type \\
Tumour site & Differentiation \\
Macroscopic type & Depth of invasion \\
Tumour size & Proximal margin details \\
Distance of tumour from & Distal margin details \\
specimen edges & \\
Histology & Circumferential margin details \\
Local invasion & Other features \\
Lymph nodes & Lymph nodes \\
Margins involved & Complete resection \\
Other pathology & TNM stage \\
Complete resection & \\
TNM stage & \\
\hline
\end{tabular}

\section{RESULTS}

One hundred and ten reports were collected from five hospitals within the network. There were 54 oesophageal reports from three hospitals and 56 gastric reports from five hospitals. Twelve oesophageal reports were excluded because there was no residual tumour after neoadjuvant treatment.

Table 2 shows the number of gastric reports that were complete, partially complete, or absent with regard to each category taken from the minimum dataset. Information about personal details and lymph node status was complete on all 56 reports, although the National Health Service number was consistently absent. Local invasion (the pT stage), histology (adenocarcinoma and the degree of differentiation), and the site of the tumour were complete in most reports $(54,52$, and 50, respectively). Only 22 reports provided an unambiguous statement regarding completeness of excision. In 46 reports, this information was provided in the main text, and from this it could be inferred whether excision was complete or not. Most reports made no mention of the $\mathrm{pM}$ component of the TNM staging, resulting in the very poor score for the pTNM category. However, it could be inferred from the totality of information provided in the report that in all cases the pM stage was pMx. With regard to the pT component of the stage, the exact numerical qualifier could be deduced for $96 \%$ of cases. The most inadequately completed category was length of specimen.

The results for the oesophageal categories were similar. Table 3 shows the number of completed categories for these reports. No report was deemed to be complete with respect to the pTNM category because the pM component was missing, as for gastric cancers.

Figure 1 shows the number of reports that were greater than $75 \%$ and less than $50 \%$ complete for both the gastric and oesophageal resection specimens. Only one oesophageal report and four gastric reports were less than 50\% complete. Seventeen oesophageal and 14 gastric reports were more than $75 \%$ complete. No report was $100 \%$ complete.

\section{DISCUSSION}

The pathology report provides crucial information, and is the main determinant of disease stage after surgery. Although, in general, the standard of reporting for oesophageal and gastric cancer specimens was good, no report for either gastric or oesophageal resection specimens was $100 \%$ complete when compared with the minimum datasets provided by the Royal College of Pathologists. Residual disease at surgery, depth of invasion, and lymph node status are the most important independent prognostic factors ${ }^{4}$ for oesophageal cancer. The pT stage and lymph node status were consistently reported, but reporting of completeness of resection was less consistent. The minimum dataset has a yes/no tick box for complete resection at all margins, and so an unambiguous statement declaring the completeness of surgical excision within the text was deemed necessary. It could be argued that the pathologist could ascertain this information from descriptions of the margins, but the pathology report has to be easily interpreted by a range of individuals involved in the care of the patient.

The circumferential margin, another independent prognostic variable for oesophageal cancer, ${ }^{5}$ was poorly completed, with only about a quarter of reports obtaining a complete score for this parameter. This was partly because of textual ambiguities and partly the result of omission. The omission of a pM stage must largely reflect the information provided to the histopathologist when resected specimens are submitted.

With both gastric and oesophageal reports the categories that were complete least often were those that required several specific parameters. For example, tumour size on the gastric reports required the tumour width, length, and thickness, and gross description on the oesophageal reports required the length of the oesophagus and stomach, the width and length of the tumour, the distances from the margins, and the macroscopic type. The high partially complete score for these categories reflects that it is inconsistency that is responsible for substandard reporting. These results are in keeping with previous audits, which have found

\begin{tabular}{|c|c|c|c|}
\hline Category on minimum dataset & $\begin{array}{l}\text { No complete } \\
\text { (\%) }\end{array}$ & $\begin{array}{l}\text { No partially } \\
\text { complete (\%) }\end{array}$ & No absent (\%) \\
\hline Personal details & $56(100)$ & $0(0)$ & $0(0)$ \\
\hline Total number of lymph nodes & $56(100)$ & $0(0)$ & $0(0)$ \\
\hline Local invasion (pT) & $54(96)$ & $2(4)$ & $0(0)$ \\
\hline $\begin{array}{l}\text { Histology (adenocarcinoma/degree of } \\
\text { differentiation) }\end{array}$ & $52(93)$ & $4(7)$ & $0(0)$ \\
\hline Site of tumour & $50(89)$ & $2(4)$ & $4(7)$ \\
\hline Margins involved & $46(82)$ & $9(16)$ & $1(2)$ \\
\hline Macroscopic type of tumour & $45(80)$ & $2(4)$ & $9(16)$ \\
\hline Type of specimen & 41 (73) & $14(25)$ & $1(2)$ \\
\hline Other pathology & $41(73)$ & $2(4)$ & $13(23)$ \\
\hline Complete resection & $22(39)$ & $4(7)$ & $30(54)$ \\
\hline Size of tumour & $21(37)$ & $33(59)$ & $2(4)$ \\
\hline Distance of tumour from edges of specimen & $17(30)$ & $18(32)$ & $21(38)$ \\
\hline TNM staging & $11(20)$ & $26(46)$ & $19(34)$ \\
\hline Length of specimen & $10(18)$ & $46(82)$ & $0(0)$ \\
\hline
\end{tabular}




\begin{tabular}{|c|c|c|c|}
\hline \multicolumn{4}{|l|}{$\begin{array}{l}\text { Table } 3 \\
(n=54)\end{array}$} \\
\hline Category on minimum dataset & $\begin{array}{l}\text { No complete } \\
\text { (\%) }\end{array}$ & $\begin{array}{l}\text { No partially } \\
\text { complete (\%) }\end{array}$ & No absent (\%) \\
\hline Personal details & $54(100)$ & $0(0)$ & $0(0)$ \\
\hline Total number of lymph nodes & $54(100)$ & $0(0)$ & $0(0)$ \\
\hline Depth of invasion & $54(100)$ & $0(0)$ & $0(0)$ \\
\hline Type of tumour & $53(98)$ & $0(0)$ & $1(2)$ \\
\hline Degree of differentiation & $46(86)$ & $0(0)$ & $8(14)$ \\
\hline Proximal margin (clear or involved) & $45(83)$ & $0(0)$ & $9(17)$ \\
\hline Distal margin (clear or involved) & $44(81)$ & $0(0)$ & $10(19)$ \\
\hline Complete resection & $27(50)$ & $1(2)$ & $26(48)$ \\
\hline $\begin{array}{l}\text { Other features (Barretts metaplasia/vascular } \\
\text { invasion) }\end{array}$ & $26(48)$ & $23(43)$ & $5(9)$ \\
\hline Gross description of tumour & $21(38)$ & $33(62)$ & $0(0)$ \\
\hline Circumferential margin & $13(24)$ & $15(28)$ & $26(48)$ \\
\hline TNM staging (pTpNpM) & $0(0)$ & $45(83)$ & $9(17)$ \\
\hline
\end{tabular}

inconsistency with reporting for colorectal cancer ${ }^{1}$ and upper gastrointestinal cancer. ${ }^{2}$

The traditional style of free text reporting allows for ambiguities to be left in and for specific points to be missed out. The minimum datasets are presented as sets of statements against which tick boxes, numbers, or short statements should be appended. The format of these documents is such that the data are not readily transferable to most computer pathology systems. In one hospital in our network, a separate proforma on a printed form was produced for each case, but this was not recorded electronically and therefore could not be retrieved for the purpose of our audit. In any case, there is a general reluctance by histopathologists to replace free text reports with proformas because they do not allow for flexibility. We are now introducing proformas for gastric and oesophageal cancers in one hospital in our network in which, for each report item, a statement is appended instead of a box being ticked; it is anticipated that most histopathologists will find this format more user friendly.

"The traditional style of free text reporting allows for ambiguities to be left in and for specific points to be missed out ${ }^{\prime \prime}$

There are also several ambiguities within the minimum datasets issued by the Royal College of Pathologists. For example, in the oesophageal cancer dataset the type of junctional tumour (that is, 1,2 , or 3 ) is not recorded. ${ }^{3}$ Serosal

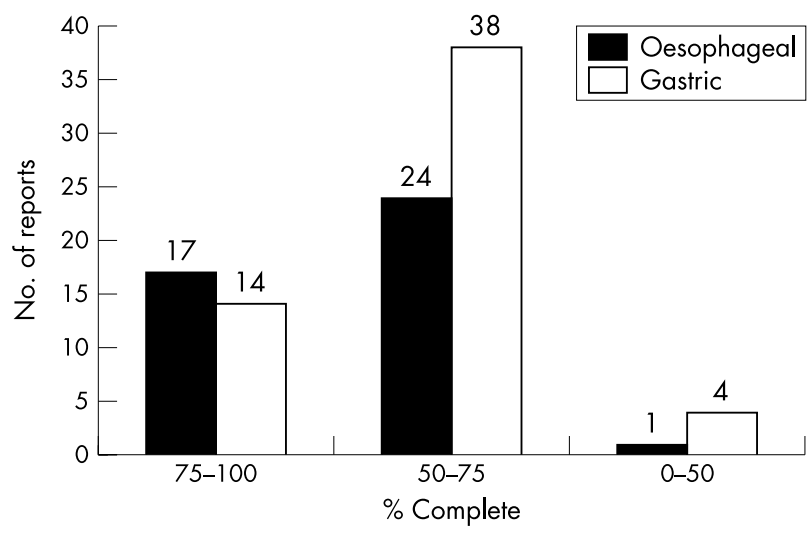

Figure 1 Number of oesophageal and gastric cancer reports complete for all categories in the dataset.

\section{Take home messages}

- We recommend the use of a standard proforma for reporting upper gastrointestinal cancers based on a minimum dataset provided by the Royal College of Pathologists to improve the quality and consistency of pathology reporting

- Modifications are needed to allow for specimens with no tumour after neoadjuvant treatment

involvement in the Royal College of Pathologists' dataset refers to the gastric portion of the tumour, which may be confusing. Tumour clearance at the deep adventitial margin should be provided in terms of distance to avoid confusion and also because of its potential prognostic relevance. ${ }^{5}$ For gastric carcinomas, vascular invasion may be of prognostic relevance, yet this is omitted in the gastric dataset.

Neoadjuvant treatment is increasingly being used before surgery. The final pathological staging in these cases should take this into account and include the prefix y (ypTNM), in addition to an indication of the response to the treatment, such as the Mandard score. ${ }^{6}$ Although it is not clear whether downstaging is associated with an improvement in prognosis, this information is important to the clinician when counselling the patient.

Proforma reporting has been shown to improve the quality and consistency of pathology reporting. ${ }^{78}$ Minimum datasets provide a framework on which cancer networks should build to provide a comprehensive proforma for the reporting of gastric and oesophageal cancers.

\section{ACKNOWLEDGEMENTS}

We thank the pathologists and surgeons in each of the hospitals for allowing us to use pathology reports from patients under their care.

\section{Authors' affiliations}

P M King, Department of Surgery, East Somerset NHS Trust, Higher Kingston, Yeovil, Somerset BA21 4AT, UK

J M Blazeby, University Department of Social Medicine and Division of Surgery, United Bristol NHS Trust, Bristol Royal Infirmary, Bristol BS2 $8 \mathrm{HW}, \mathrm{UK}$

J Gupta, Department of Histopathology, North Bristol NHS Trust, Southmead, Bristol BS10 5NB, UK

D Alderson, Division of Surgery, United Bristol NHS Trust, Bristol Royal Infirmary, Bristol BS2 8HW, UK

M Moorghen, Department of Histopathology, United Bristol NHS Trust 


\section{REFERENCES}

1 Bull AD, Biffin AH, Mella J, et al. Colorectal cancer pathology reporting: a regional audit. J Clin Pathol 1997:50:138-42.

2 Burroughs SH, Biffin AH, Pye JK, et al. Oesophageal and gastric cancer pathology reporting: a regional audit. J Clin Pathol 1999;52:435-9.

3 Royal College of Pathologists. Minimum datasets for oesophageal and gastric cancer pathology reporting (www.rcpath.org.uk, 2003).

4 Ibrahim NB. ACP Best Practice No 155. Guidelines for handling oesophageal biopsies and resection specimens and their reporting. J Clin Pathol 2000;53:89-94.
5 Dexter SP, Sue-Ling H, McMahon MJ, et al. Circumferential resection margin involvement: an independent predictor of survival following surgery for oesophageal cancer. Gut 2001;48:667-70.

6 Mandard AM, Dalibard F, Mandard JC, et al. Pathologic assessment of tumor regression after preoperative chemoradiotherapy of esophageal carcinoma. Clinicopathologic correlations. Cancer 1994;73:2680-6.

7 Rigby K, Brown SR, Lakin G, et al. The use of a proforma improves colorectal cancer pathology reporting. Ann R Coll Surg Engl 1999;81:401-3.

8 Mathers ME, Shrimankar J, Scott DJ, et al. The use of a standard proforma in breast cancer reporting. J Clin Pathol 2001;54:809-1 1 .

\section{Clinical Evidence-Call for contributors}

Clinical Evidence is a regularly updated evidence based journal available worldwide both as a paper version and on the internet. Clinical Evidence needs to recruit a number of new contributors. Contributors are health care professionals or epidemiologists with experience in evidence based medicine and the ability to write in a concise and structured way.

\section{Currently, we are interested in finding contributors with an interest in} the following clinical areas:

Altitude sickness; Autism; Basal cell carcinoma; Breast feeding; Carbon monoxide poisoning; Cervical cancer; Cystic fibrosis; Ectopic pregnancy; Grief/bereavement; Halitosis; Hodgkins disease; Infectious mononucleosis (glandular fever); Kidney stones; Malignant melanoma (metastatic); Mesothelioma; Myeloma; Ovarian cyst; Pancreatitis (acute); Pancreatitis (chronic); Polymyalgia rheumatica; Post-partum haemorrhage; Pulmonary embolism; Recurrent miscarriage; Repetitive strain injury; Scoliosis; Seasonal affective disorder; Squint; Systemic lupus erythematosus; Testicular cancer; Varicocele; Viral meningitis; Vitiligo However, we are always looking for others, so do not let this list discourage you.

\section{Being a contributor involves:}

- Appraising the results of literature searches (performed by our Information Specialists) to identify high quality evidence for inclusion in the journal.

- Writing to a highly structured template (about 2000-3000 words), using evidence from selected studies, within 6-8 weeks of receiving the literature search results.

- Working with Clinical Evidence Editors to ensure that the text meets rigorous epidemiological and style standards.

- Updating the text every eight months to incorporate new evidence.

- Expanding the topic to include new questions once every 12-18 months.

If you would like to become a contributor for Clinical Evidence or require more information about what this involves please send your contact details and a copy of your CV, clearly stating the clinical area you are interested in, to Claire Folkes (cfolkes@bmigroup.com).

\section{Call for peer reviewers}

Clinical Evidence also needs to recruit a number of new peer reviewers specifically with an interest in the clinical areas stated above, and also others related to general practice. Peer reviewers are health care professionals or epidemiologists with experience in evidence based medicine. As a peer reviewer you would be asked for your views on the clinical relevance, validity, and accessibility of specific topics within the journal, and their usefulness to the intended audience (international generalists and health care professionals, possibly with limited statistical knowledge). Topics are usually 2000-3000 words in length and we would ask you to review between 2-5 topics per year. The peer review process takes place throughout the year, and our turnaround time for each review is ideally 10-14 days.

If you are interested in becoming a peer reviewer for Clinical Evidence, please complete the peer review questionnaire at www.clinicalevidence.com or contact Claire Folkes(cfolkes@bmigroup.com). 